\title{
Glycemic effects of simvastatin: Where do we stand?
}

\author{
Nor Razida Razali ${ }^{1}$, Hasniza Zaman Huri ${ }^{1,2}$, Luqman Ibrahim ${ }^{3}$, Shireene Ratna Vethakkan ${ }^{3}$, \\ Bashar Mudhaffar Abdullah ${ }^{2}$
}

\begin{abstract}
${ }^{1}$ Department of Pharmacy, Faculty of Medicine, University of Malaya, Kuala Lumpur, Malaysia, ${ }^{2}$ Clinical Investigation Centre, University Malaya Medical Centre, Kuala Lumpur, Malaysia, ${ }^{3}$ Department of Medicine, Faculty of Medicine, University of Malaya, Kuala Lumpur, Malaysia
\end{abstract}

\begin{abstract}
In clinical practice, simvastatin is usually used in the treatment of dyslipidemia patients and those at risk of or with established cardiovascular disease. However, previous studies have shown that simvastatin has the potential to affect glycemic parameters as it reportedly reduced insulin secretion and sensitivity. The exact mechanism by which simvastatin affects glycemia is still unknown, but previous studies have postulated the involvement of the glucose-insulin secretion mechanism. This review focuses on the effects of simvastatin, either alone or in combination with other lipid lowering agents, antidiabetics and antihypertensives, on glucose homeostasis. Some studies have reported that simvastatin might impair the levels of glucose metabolism markers in the blood while others have reported no effect or improvement in glycemia.
\end{abstract}

Keywords: Simvastatin/effects. Glucose. Insulin secretion. Insulin sensitivity. Diabetes. Concurrent medications.

\section{INTRODUCTION}

Statins or 3-hydroxyl-3 methylglutaryl co-enzyme A (HMG-CoA) reductase inhibitors are used worldwide to treat dyslipidemia and also as part of the management of patients who have high risk of developing or established cardiovascular events resulting from type 2 diabetes mellitus (T2DM) or hypertension (Grover, Luthra, Maroo, 2014; Perreault et al., 2009). T2DM patients have a twoto four-fold increase in risk of cardiovascular disease as compared to the general population (Colhoun et al., 2004). In addition, these patients tend to have high levels of triglyceride, low levels of high-density lipoprotein (HDL) with smaller and denser low-density lipoprotein (LDL) particles that promote atherogenesis (Vijan, Hayward, 2004).

The key action of statins are by inhibition of the $\mathrm{HMG}-\mathrm{CoA}$ reductase enzyme, hence reducing mevalonate synthesis and subsequently inhibits several other isoprenoid pathways as well as cholesterol synthesis (Gazzerro et al., 2012; Sirtori, 2014) (Figure 1). Currently,

\footnotetext{
*Correspondence: Hasniza Zaman Huri. Department of Pharmacy, Faculty of Medicine, University of Malaya, Kuala Lumpur, Malaysia. E-mail: hasnizazh@um.edu.my
}

there are several types of statins available in the market, such as simvastatin, atorvastatin, lovastatin, fluvastatin, rosuvastatin and pravastatin. Cerivastatin has been withdrawn from the market after 52 deaths were reported due to kidney failure as a result of rhabdomyolysis (Furberg, Pitt, 2001).

In the United States, data from the National Health and Nutrition Examination Survey 2011-2012 showed that among adults aged 40 years and above who were using lipid-lowering drugs, $83 \%$ were using a statin, $10 \%$ a combination of a statin and a non-statin and 7\% nonstatin. Simvastatin was the most commonly used statin $(42 \%)$, followed by atorvastatin $(20.2 \%)$, pravastatin $(11.2 \%)$, rosuvastatin $(8.2 \%)$ and lovastatin $(7.4 \%)(\mathrm{Gu}$ et al., 2015).

Simvastatin or its brand name Zocor (Al-Foraih, Somerset, 2016) is one of the most commonly used statins because of its effectiveness in reducing LDL cholesterol levels, produces fewer adverse effects, and is more affordable compared with other statins. Simvastatin is a semi-synthetic derivative of lovastatin which is obtained from a fermented product of Aspergillus terreus (Manzoni, Rollini, 2002). Most patients are prescribed simvastatin at dosages of 10, 20, or $40 \mathrm{mg} /$ day. However, the use of simvastatin at $80 \mathrm{mg} /$ day is restricted because of a high 


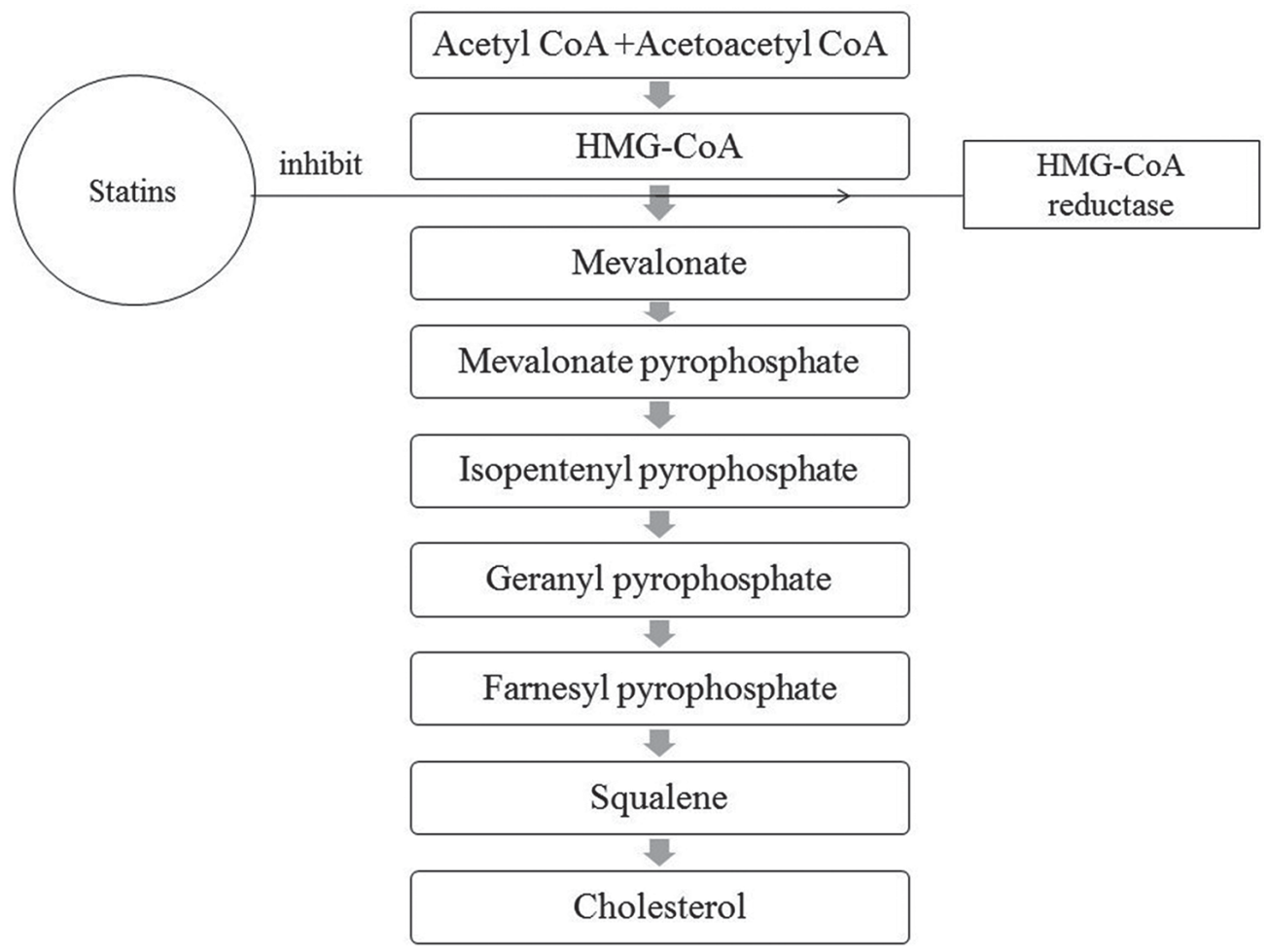

FIGURE 1 - Inhibition of the cholesterol synthesis pathway by statins.

risk of muscle injury (FDA, 2011).

The efficacy of simvastatin in reducing the risk, morbidity, and mortality of cardiovascular events has been demonstrated in various studies, such as the Scandinavian Simvastatin Survival Study (Pedersen et al., 1998), the Heart Protection Study (Heart Protection Study Collaborative Group, 2002), the Study of the Effectiveness of Additional Reduction in Cholesterol and Homocysteine (SEARCH) Collaborative Group (Meade et al., 2010) and others (Ceriello, 2002; Dobs et al., 2008; Foody et al., 2008). However, some studies have reported association between statins and glycemia, but such effects are controversial and conflicting ranging from adverse, neutral to beneficial. In diabetics, simvastatin has been shown to worsen glycemic control and insulin secretion (Bellia et al., 2012), improve insulin resistance (Paolisso et al., 2000 ) or to have no effect on glucose levels (Farrer et al., 1994; Szendroedi et al., 2009).

In terms of solubility, statins can be classified into water-soluble (hydrophilic) and lipid soluble (lipophilic). Atorvastatin, fluvastatin, lovastatin, and simvastatin are lipophilic statins, while rosuvastatin and pravastatin are hydrophilic statins (Igel, Sudhop, Bergmann, 2002). For lipophilic statins, it can diffuse through the plasma membranes of extrahepatic cells (for example beta cells, adipocytes and skeletal muscle cells), which can result in a diabetogenic effect (Aiman, Najmi, Khan, 2014;
Schachter, 2005). As simvastatin is a lipophilic statin, it has the potential to reduce insulin secretion and sensitivity (Koh et al., 2009).

\section{Effect of simvastatin on glucose metabolism: in vitro studies}

The exact mechanisms underlying the effect of simvastatin on glycemia are still unknown. However, previous studies have implicated the inhibition of glucose-stimulated insulin secretion. Several experimental studies have indicated how simvastatin affects glucose metabolism (Figure 2).

The effect of statins (simvastatin, simvastatin acids, and pravastatin) on $\beta$ cell function has been investigated in rat pancreatic $\beta$ cells. Cytosolic calcium $\left(\mathrm{Ca}^{2+}\right)$ concentration is an important component in the regulation of pancreatic $\beta$ cells (De Marchi et al., 2014). A reduction in the cytosolic $\mathrm{Ca}^{2+}$ concentration leads to impairment of insulin secretion. In the study by Yada et al. (1999) has demonstrated that simvastatin inhibited $\beta$ cell L-type $\mathrm{Ca}^{2+}$ channels and reduced insulin secretion but pravastatin did not. After administration of simvastatin for 20 seconds, L-arginine and potassium chloride-induced insulin release were inhibited (Yada et al., 1999).

The mechanisms by which simvastatin impairs insulin secretion have been elucidated using mouse islet 
$\boldsymbol{\beta}$ cell lines, MIN6. Compared to normal control cells, simvastatin significantly inhibited insulin secretion in a dose-dependent manner. The inhibition of insulin secretion was indirectly caused by reduced levels of glucose transporter 2 (GLUT2). Simvastatin reduced the adenosine triphosphate (ATP) levels in MIN6 cells, increased the ATP-sensitive potassium channel (KATP) current and reduced the L-type - $\mathrm{Ca}^{2+}$ current. Simvastatin may also reduce insulin secretion by increasing the rectifier potassium channel (Kir6.2) current while simultaneously decreasing the voltage-dependent $\mathrm{Ca}^{2+}$ channel 1.2 (Cav1.2) current, which leads to inhibition of membrane cell depolarization and inhibition of calcium influx (Zhou et al., 2014).

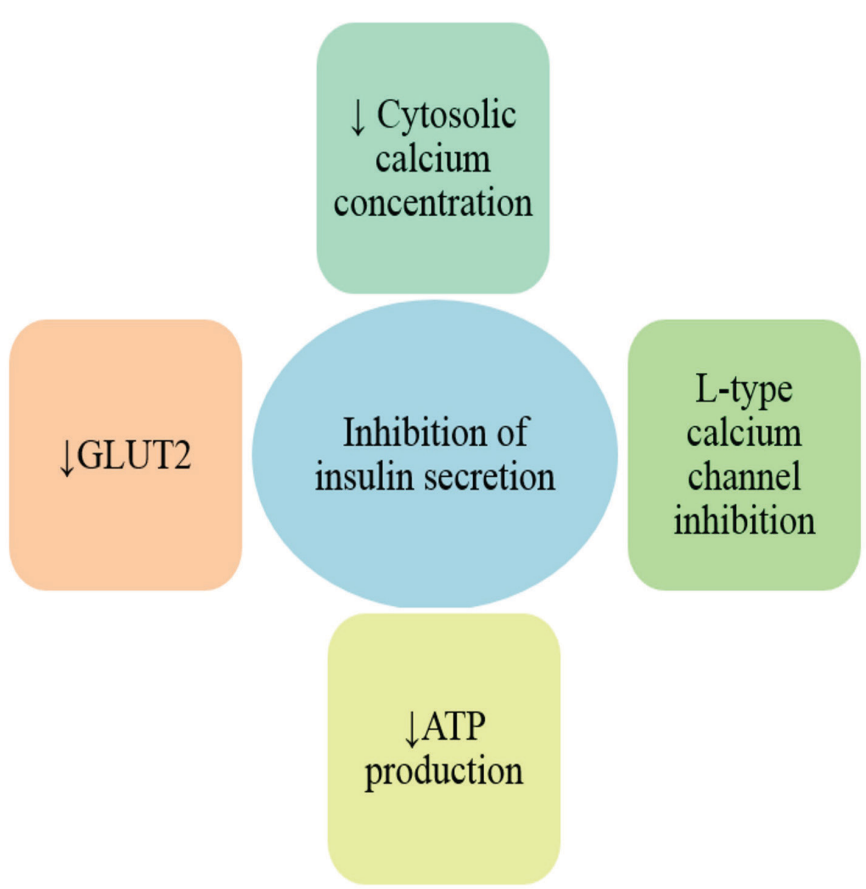

FIGURE 2 - Previous experimental findings on the effect of simvastatin on glucose-insulin secretion.

\section{Glycemic effect of simvastatin: human data}

Simvastatin has been reported to increase plasma glucose levels and reduce insulin sensitivity. A few studies have measured the effect of simvastatin treatment on glucose homeostasis (Table I). In a study by Koh et al. (2008) reported that simvastatin improved flow-mediated dilation, but reduced adiponectin levels and insulin sensitivity in hypercholesterolemia patients (Koh et al., 2008). Patients who were on simvastatin $80 \mathrm{mg}$ /day had a $7 \%$ increase in mean plasma glucose levels after 2 months of treatment. Meanwhile, those who were on simvastatin $10,20,40$, or $80 \mathrm{mg}$ /day had increased insulin secretions relative to the baseline after 2 months of treatment, which is indicative of deterioration in insulin sensitivity. The same study also demonstrated that there was a slight reduction in insulin sensitivity in the simvastatintreated group, as measured using the quantitative insulin sensitivity check index (QUICKI) (Koh et al., 2008). A separate study by the same authors showed significant reductions in insulin sensitivity and plasma adiponectin levels in hypercholesterolemia patients after taking simvastatin $20 \mathrm{mg}$ /day for 2 months. However, there were no significant differences in insulin or glucose levels compared to baseline (Koh et al., 2015).

A study in which patients were selected randomly to receive either simvastatin $20 \mathrm{mg}$ /day or rosuvastatin $20 \mathrm{mg} /$ day showed that there was no effect of simvastatin on insulin sensitivity and glycemic control after 4 weeks of treatment (Bellia et al., 2010). However, another study by the same authors reported that the simvastatin and rosuvastatin treatments worsen fasting blood glucose (FBG) and A1C levels after 12 months without affecting insulin sensitivity (Bellia et al., 2012). On the same note, a study by Sen et al. (2002) found that in simvastatin group, A1C levels were significantly increased at follow-up at 90 and 180 days compared to day 1 (Sen et al., 2002).

Conversely, some studies have reported a lack of association between simvastatin treatment and blood glucose levels. After 90 days of simvastatin treatment, the homeostasis model assessment - insulin resistance (HOMA-IR) values and FBG levels remained unchanged in patients with isolated hypercholesterolemia, even though there were improvements in plasma lipid levels (Krysiak, Okopien, 2013a). As for T2DM patients, some studies have reported no significant changes in glucose parameters after simvastatin treatment (Farrer et al.,1994; Hwu et al., 1999). Szendroedi et al. (2009) reported that there was no effect of simvastatin on insulin sensitivity, fasting insulin levels or HOMA-B levels (Szendroedi et al., 2009). In addition, a study by Hydrie et al. (2007) found after receiving simvastatin for 3 months, there were no significant differences in HOMA-IR values compared to baseline. However, 20 patients with T2DM who were having insulin resistance with HOMA-IR values of more than 2.8 at the beginning of the study demonstrated improvements in insulin sensitivity after receiving simvastatin (Hydrie et al., 2007).

\section{Simvastatin and new-onset Diabetes}

The Heart Protection Study has suggested that there was no association between simvastatin use and new-onset diabetes, although previous studies have reported that statins 
TABLE I - The effect of simvastatin on glucose metabolism markers in human data

\begin{tabular}{|c|c|c|c|c|c|c|c|c|c|c|}
\hline \multirow[b]{2}{*}{ Study design } & \multirow[b]{2}{*}{ Country } & \multirow[b]{2}{*}{ Subjects } & \multirow[b]{2}{*}{$\mathbf{N}$} & \multirow{2}{*}{$\begin{array}{l}\text { Mean } \\
\text { follow-up }\end{array}$} & \multirow[b]{2}{*}{ Method } & \multicolumn{5}{|c|}{ Outcome (compared with baseline) } \\
\hline & & & & & & A1C & Glucose & Insulin & $\begin{array}{c}\text { Insulin } \\
\text { sensitivity }\end{array}$ & Adiponectin \\
\hline $\begin{array}{l}\text { Randomized } \\
\text {, double } \\
\text { blind, placebo } \\
\text { controlled } \\
\text { parallel study } \\
\text { (Koh et al., } \\
\text { 2008) }\end{array}$ & Korea & $\begin{array}{l}\text { Hypercholes- } \\
\text { terolemia }\end{array}$ & 156 & 2 months & $\begin{array}{c}\text { Each } 32 \text { patients given } \\
\text { either placebo, SIM 10, } \\
20,40 \text { or } 80 \mathrm{mg} / \text { day }\end{array}$ & $\begin{array}{l}\text { Not } \\
\text { measured }\end{array}$ & $\begin{array}{l}\text { SIM } 80 \mathrm{mg} / \\
\text { day increase } \\
\text { glucose level }\end{array}$ & $\begin{array}{l}\text { SIM } 10,20, \\
40 \text { and } \\
80 \mathrm{mg} / \text { day } \\
\text { increase } \\
\text { insulin level }\end{array}$ & $\begin{array}{l}\text { SIM } 10,20 \\
40 \text { and } 80 \\
\text { mg/day } \\
\text { reduce insulin } \\
\text { sensitivity }\end{array}$ & $\begin{array}{l}\text { SIM } 10,20,40 \\
\text { and } 80 \mathrm{mg} / \text { day } \\
\text { decrease } \\
\text { plasma } \\
\text { adiponectin }\end{array}$ \\
\hline $\begin{array}{l}\text { Randomized } \\
\text {, single-blind } \\
\text {, placebo- } \\
\text { controlled, } \\
\text { parallel study } \\
\text { (Koh et al., } \\
\text { 2015) } \\
\end{array}$ & Korea & $\begin{array}{l}\text { Hypercholes- } \\
\text { terolemia }\end{array}$ & 203 & 2 months & $\begin{array}{c}\text { Each } 51 \text { patients receive } \\
\text { either placebo, EZE } \\
10 \mathrm{mg}+\text { SIM } 10 \mathrm{mg} \\
\text { (Vyto10), EZE } 10 \mathrm{mg}+ \\
\text { SIM } 20 \mathrm{mg} \text { (Vyto20) or } \\
\text { SIM } 20 \mathrm{mg} \text { alone once } \\
\text { daily }\end{array}$ & $\begin{array}{l}\text { SIM } 20 \text { mg no } \\
\text { different }\end{array}$ & $\begin{array}{l}\text { SIM } 20 \\
\text { mg no } \\
\text { different }\end{array}$ & $\begin{array}{l}\text { SIM } \\
20 \mathrm{mg} \\
\text { group not } \\
\text { significant } \\
\text { change } \\
\text { insulin } \\
\text { level }\end{array}$ & $\begin{array}{l}\text { SIM } 20 \\
\text { mg group } \\
\text { reduce the } \\
\text { insulin } \\
\text { sensitivity }\end{array}$ & $\begin{array}{c}\text { SIM 20 } \\
\text { mg group } \\
\text { significantly } \\
\text { reduce } \\
\text { plasma } \\
\text { adiponectin } \\
\text { level } \\
\end{array}$ \\
\hline $\begin{array}{l}\text { Randomized, } \\
\text { single-blind, } \\
\text { parallel } \\
\text { intervention } \\
\text { study (Bellia } \\
\text { et al., 2010) }\end{array}$ & Italy & $\begin{array}{l}\text { Patients with } \\
\text { middle aged } \\
\text { with T2DM and } \\
\text { mild treated } \\
\text { dyslipidemia }\end{array}$ & 29 & 4 weeks & $\begin{array}{l}\text { Patients receive either } \\
\text { ROS } 20 \mathrm{mg} / \text { day or SIM } \\
20 \mathrm{mg} / \text { day }\end{array}$ & $\begin{array}{l}\text { Not } \\
\text { measured }\end{array}$ & $\begin{array}{l}\text { No effect } \\
\text { in both } \\
\text { groups }\end{array}$ & $\begin{array}{l}\text { No effect } \\
\text { in both } \\
\text { groups }\end{array}$ & $\begin{array}{l}\text { No effect } \\
\text { in both } \\
\text { groups }\end{array}$ & $\begin{array}{l}\text { No effect in } \\
\text { both groups }\end{array}$ \\
\hline $\begin{array}{l}\text { Randomized, } \\
\text { single-blind } \\
\text { with two period } \\
\text { (Bellia } \text { et al., } \\
\text { 2012) }\end{array}$ & Italy & $\begin{array}{l}\text { Well controlled } \\
\text { T2DM patients }\end{array}$ & 27 & 12 months & $\begin{array}{l}\text { Patients receive either } \\
\text { ROS } 20 \mathrm{mg} \text { /day or SIM } \\
20 \mathrm{mg} \text { /day for } 6 \text { months } \\
\text { and switch the treatment } \\
\text { for following next } 6 \\
\text { months }\end{array}$ & $\begin{array}{l}\text { Both groups } \\
\text { worsen A1C }\end{array}$ & $\begin{array}{l}\text { Both } \\
\text { groups } \\
\text { increase } \\
\text { FBG }\end{array}$ & $\begin{array}{l}\text { No } \\
\text { changes }\end{array}$ & $\begin{array}{l}\text { No effect } \\
\text { in both } \\
\text { groups }\end{array}$ & $\begin{array}{l}\text { Not } \\
\text { significant } \\
\text { increase }\end{array}$ \\
\hline $\begin{array}{l}\text { Double blind } \\
\text { randomized } \\
\text { placebo- } \\
\text { controlled study } \\
\text { (Sen et a.l., } \\
\text { 2002) } \\
\end{array}$ & India & $\begin{array}{l}\text { T1DM and T2DM } \\
\text { with diabetic } \\
\text { retinopathy }\end{array}$ & 50 & 180 days & $\begin{array}{c}\text { Patients receive } \\
\text { either SIM } 20 \mathrm{mg} / \text { day } \\
\text { or placebo }\end{array}$ & $\begin{array}{l}\text { A1C in SIM } \\
\text { group increase } \\
\text { throughout the } \\
\text { follow-up at } \\
90 \text { days and } \\
180 \text { days } \\
\end{array}$ & $\begin{array}{l}\text { No } \\
\text { significant } \\
\text { changes in } \\
\text { FBG }\end{array}$ & $\begin{array}{l}\text { No } \\
\text { significant } \\
\text { changes }\end{array}$ & $\begin{array}{l}\text { Not } \\
\text { measured }\end{array}$ & $\begin{array}{l}\text { Not } \\
\text { measured }\end{array}$ \\
\hline $\begin{array}{l}\text { Randomized } \\
\text { study } \\
\text { (Tsutamoto } \\
\text { et al., 2009) }\end{array}$ & Japan & $\begin{array}{l}\text { Non-ischemic } \\
\text { chronic heart } \\
\text { failure }\end{array}$ & 71 & $\begin{array}{l}2.2 \pm 0.15 \\
\text { years }\end{array}$ & $\begin{array}{l}\text { Patients receive either } \\
\text { SIM } 5 \mathrm{mg} / \text { day } \\
(\mathrm{n}=35) \\
\text { or ROS } 2.5 \mathrm{mg} / \text { day } \\
(\mathrm{n}=36)\end{array}$ & $\begin{array}{c}\text { Slightly } \\
\text { increase in } \\
\text { SIM group } \\
\text { \& decrease in } \\
\text { rosuvastatin } \\
\text { group } \\
\end{array}$ & $\begin{array}{l}\text { Not } \\
\text { measured }\end{array}$ & $\begin{array}{l}\text { Not } \\
\text { measured }\end{array}$ & $\begin{array}{l}\text { Not } \\
\text { measured }\end{array}$ & $\begin{array}{l}\text { No changes } \\
\text { in SIM } \\
\text { group but } \\
\text { increase in } \\
\text { ROS group }\end{array}$ \\
\hline $\begin{array}{l}\text { Randomized, } \\
\text { case-control } \\
\text { study (Krysiak, } \\
\text { Okopien, } \\
\text { 2013a) } \\
\end{array}$ & Polland & $\begin{array}{c}\text { Isolated } \\
\text { hypertriglyceri- } \\
\text { demia }\end{array}$ & 39 & 3 months & $\begin{array}{l}\text { Patients receive placebo } \\
\text { or SIM } 40 \mathrm{mg} / \text { day }\end{array}$ & $\begin{array}{l}\text { Not } \\
\text { measured }\end{array}$ & $\begin{array}{l}\text { Both } \\
\text { groups not } \\
\text { significant }\end{array}$ & $\begin{array}{l}\text { Not } \\
\text { measured }\end{array}$ & $\begin{array}{l}\text { Both } \\
\text { groups not } \\
\text { significant }\end{array}$ & $\begin{array}{l}\text { Not } \\
\text { measured }\end{array}$ \\
\hline $\begin{array}{l}\text { Double blind } \\
\text { placebo } \\
\text { controlled } \\
\text { study (Farrer } \\
\text { et al., 1994) } \\
\end{array}$ & $\begin{array}{l}\text { United } \\
\text { Kingdom }\end{array}$ & $\begin{array}{c}\text { Patients } \\
\text { with T2DM } \\
\text { dyslipidemia and } \\
\text { mild hypertriglyce- } \\
\text { ridemia }\end{array}$ & 70 & 6 months & $\begin{array}{l}\text { Patients randomized to } \\
\text { receive placebo or SIM }\end{array}$ & $\begin{array}{l}\text { No } \\
\text { significant } \\
\text { changes }\end{array}$ & $\begin{array}{l}\text { No } \\
\text { significant } \\
\text { changes }\end{array}$ & $\begin{array}{l}\text { No } \\
\text { significant } \\
\text { changes }\end{array}$ & $\begin{array}{l}\text { Not } \\
\text { measured }\end{array}$ & $\begin{array}{l}\text { Not } \\
\text { measured }\end{array}$ \\
\hline $\begin{array}{l}\text { Randomized, } \\
\text { double-blind, } \\
\text { placebo- } \\
\text { controlled and } \\
\text { two-period } \\
\text { crossover study } \\
\text { (Hwu et al., } \\
1999 \text { ) }\end{array}$ & Taiwan & $\begin{array}{c}\text { Patient T2DM } \\
\text { with hypercholes- } \\
\text { terolemia }\end{array}$ & 19 & 6 months & $\begin{array}{l}\text { Patients receive either } \\
\text { SIM } 20 \mathrm{mg} \text { /day or } \\
\text { placebo for } 3 \text { months and } \\
\text { exchange the treatment } \\
\text { for subsequent } 3 \text { months }\end{array}$ & $\begin{array}{l}\text { No effect } \\
\text { in SIM } \\
\text { group }\end{array}$ & $\begin{array}{l}\text { No effect } \\
\text { in SIM } \\
\text { group }\end{array}$ & $\begin{array}{l}\text { Not } \\
\text { measured }\end{array}$ & $\begin{array}{l}\text { No effect } \\
\text { in SIM } \\
\text { group }\end{array}$ & $\begin{array}{l}\text { Not } \\
\text { measured }\end{array}$ \\
\hline $\begin{array}{l}\text { Randomized, } \\
\text { double-blind, } \\
\text { placebo- } \\
\text { controlled, } \\
\text { single } \\
\text { center study } \\
\text { (Szendroedi et } \\
\text { al., 2009) }\end{array}$ & German & $\begin{array}{l}\text { Non-obese T2DM } \\
\text { patients }\end{array}$ & 30 & 2 months & $\begin{array}{l}\text { Patients given placebo or } \\
\text { SIM } 80 \mathrm{mg} \text { /day }\end{array}$ & $\begin{array}{l}\text { No significant } \\
\text { changes }\end{array}$ & $\begin{array}{l}\text { Not } \\
\text { measured }\end{array}$ & $\begin{array}{l}\text { No } \\
\text { significant } \\
\text { changes }\end{array}$ & $\begin{array}{l}\text { No } \\
\text { significant } \\
\text { changes }\end{array}$ & $\begin{array}{l}\text { Not } \\
\text { measured }\end{array}$ \\
\hline $\begin{array}{l}\text { Randomized, } \\
\text { case control } \\
\text { study (Hydrie et } \\
\text { al., 2007) }\end{array}$ & Pakistan & $\begin{array}{l}\text { Patients with } \\
\text { T2DM }\end{array}$ & 100 & 3 months & $\begin{array}{c}50 \text { patients receive SIM } \\
40 \mathrm{mg} / \text { day as case and } \\
50 \text { patients as control } \\
\text { group }\end{array}$ & Not measured & $\begin{array}{l}\text { No } \\
\text { significant } \\
\text { changes }\end{array}$ & $\begin{array}{l}\text { No } \\
\text { significant } \\
\text { changes }\end{array}$ & $\begin{array}{l}\text { No } \\
\text { significant } \\
\text { changes }\end{array}$ & $\begin{array}{l}\text { Not } \\
\text { measured }\end{array}$ \\
\hline
\end{tabular}

Abbreviation: SIM (simvastatin); EZE (ezetimibe); ROS (rosuvastatin); T1DM (type 1 diabetes mellitus); T2DM (type 2 diabetes mellitus). Significant value $\mathrm{p}<0.05$ 
might induce the new-onset of diabetes. Among 14, 573 subjects without diabetes at study entry, it was noted that there was no significant difference in number of new-onset diabetes between the simvastatin group (4.6\%) and the placebo group (4.0\%). After follow-up for 4.6 years, among 1087 subjects who had diabetes at study entry, there was no significant difference in increased A1C among treatment groups (Heart Protection Study Collaborative Group, 2003).

However, the Study of Effectiveness of Additional Reductions in Cholesterol, Homocysteine (SEARCH) trial showed that there was a slight increase in new-onset diabetes with high dose simvastatin, $80 \mathrm{mg} /$ day $(11.6 \%)$ compared to low dose, simvastatin $20 \mathrm{mg} /$ day (10.9\%) (Armitage et al., 2010) (Table II).

\section{Effect of simvastatin and concurrent medications on glycemic control}

Certain patients, like those with metabolic syndrome and T2DM, require combinations of lipid lowering drugs because the use of simvastatin alone may fail to result in optimal lipid targets. Fenofibrate and niacin are the lipid lowering agents which are most often prescribed together with statins (Cannon, 2008). However, these concomitant drugs may increase the risk of drug-drug interaction with regards to glycemic effects, as shown in Table III.

Niacin therapy is known to have beneficial effects in patients with dyslipidemia as it increases HDL cholesterol levels and at the same time reduces triglyceride and LDL cholesterol levels. However, niacin has the potential to increase blood glucose levels (Bays, 2008; Sazonov et al., 2013; Zhao et al., 2004). In a study by Vittone et al. (2007), it was found that three years' usage of niacin in combination with simvastatin had a slight adverse effect on glycemic control, whereby FBG was increased by $3 \%$, fasting insulin was elevated by $19 \%$, and insulin sensitivity was reduced by $10 \%$ compared to baseline results (Vittone et al., 2007). As such, even though niacin when used alone or in combination with a statin gives beneficial effects to T2DM patients (in terms of achievement of target lipid levels), glucose levels should be monitored in those who are on long-term treatment (Ding, Li, Wen, 2015).

In general, fibrates reduce plasma triglyceride levels by $30-50 \%$, reduce LDL cholesterol levels by up to $20 \%$

TABLE II - Comparison of relative risk of new-onset diabetes with simvastatin use

\begin{tabular}{|c|c|c|c|c|}
\hline Study's Name & Subjects & $\begin{array}{c}\text { Mean } \\
\text { follow-up }\end{array}$ & Method & $\begin{array}{c}\text { Relative risk of NOD } \\
(95 \% \mathrm{CI})\end{array}$ \\
\hline $\begin{array}{l}\text { Heart Protection Study } \\
\text { (HPS) } \\
\text { (Heart Protection } \\
\text { Study Collaborative } \\
\text { Group, 2003) } \\
\end{array}$ & $\begin{array}{c}\text { Patients with diabetes } \\
(5,963) \text { and patient } \\
\text { occlusive arterial disease } \\
\text { with non-diabetes } \\
(14,573)\end{array}$ & 4.6 years & $\begin{array}{l}\text { Patients randomized } \\
\text { to receive either } \\
\text { simvastatin } 40 \mathrm{mg} / \text { day } \\
\text { or matching placebo }\end{array}$ & $\begin{array}{c}\text { Simvastatin vs. placebo } \\
1.14(0.98-1.33)\end{array}$ \\
\hline $\begin{array}{l}\text { Study of Effectiveness } \\
\text { of Additional Reductions } \\
\text { in Cholesterol and } \\
\text { Homocysteine } \\
\text { (SEARCH) (Armitage } \\
\text { et al.,2010) }\end{array}$ & $\begin{array}{l}\text { 12, } 064 \text { patients with } \\
\text { history of myocardial } \\
\text { infarction }\end{array}$ & 6.7 years & $\begin{array}{l}\text { Patients randomized to } \\
\text { receive either low dose, } \\
\text { simvastatin } 20 \mathrm{mg} \\
(6,033 \text { patients }) \text { or high } \\
\text { dose } 80 \mathrm{mg} \\
(6,031 \text { patients }) \\
\text { daily }\end{array}$ & $\begin{array}{c}\text { High dose vs. low dose } \\
1.07(0.95-1.19)\end{array}$ \\
\hline
\end{tabular}

Abbreviation: NOD (New-onset diabetes)

TABLE III - Summary effects of simvastatin in combination with other lipid lowering medications based on previous studies

\begin{tabular}{|c|c|c|}
\hline Authors & Combination lipid lowering drug & Finding (compared to baseline) \\
\hline (Vittone et al., 2007) & Niacin + simvastatin & $\begin{array}{l}\uparrow \mathrm{FBG}, \uparrow \text { fasting insulin, } \\
\downarrow \text { insulin sensitivity }\end{array}$ \\
\hline (Derosa et al., 2009) & Fenofibrate + simvastatin & $\begin{array}{l}\downarrow \mathrm{A} 1 \mathrm{C} \text {, no significant changes in fasting } \\
\text { glucose, fasting insulin, post-prandial glucose }\end{array}$ \\
\hline (Koh et al., 2015) & Ezetimibe + simvastatin (Vyto 10$)$ & $\begin{array}{c}\downarrow \text { Fasting insulin, } \uparrow \text { adiponectin, } \\
\uparrow \text { insulin sensitivity }\end{array}$ \\
\hline
\end{tabular}


and increase HDL cholesterol levels by 5-15\% (Chapman, 2003; National Cholesterol Education Program, 2002). In contrast to niacin, fenofibrate is often used with simvastatin in T2DM patients to achieve target lipid levels because fibrates do not lead to the worsening of blood glucose levels. The simvastatin-fenofibrate combination has been shown to be significantly more effective than simvastatin alone (Grundy et al., 2005). A study whose objective was to determine the effectiveness of fenofibrate alone, simvastatin alone and both drugs combined recruited 241 patients with T2DM and dyslipidemia who have never been prescribed lipid lowering medications before. The patients were divided into three groups; one received fenofibrate $145 \mathrm{mg}$ /day, another received simvastatin 40 $\mathrm{mg} /$ day, and the remaining received a combination of the aforementioned drugs. Glucose and lipid profiles were evaluated at baseline, 6 and 12 months. As expected, total cholesterol, LDL cholesterol, and triglyceride levels decreased while HDL cholesterol increased. In patients treated with simvastatin alone, there was no difference between baseline A1C levels and those at 12 months. However, A1C levels were significantly decreased in the other two groups. After 6 and 12 months of treatment, there were no significant differences in FBG, postprandial glucose and fasting plasma insulin levels in all three groups (Derosa et al., 2009).

Ezetimibe is a LDL cholesterol-lowering drug that acts by inhibiting the absorption of dietary cholesterol in the small intestine (Ahmed, Byrne, 2010). In a randomized double-blinded study, T2DM patients received stable doses of thiazolidinediones (rosiglitazone $2-8 \mathrm{mg}$ /day or pioglitazone $15-45 \mathrm{mg} /$ day) for at least 3 months and simvastatin $20 \mathrm{mg}$ /day for 6 weeks prior to the study. Patients were then randomized to receive either ezetimibe $10 \mathrm{mg} /$ day $(\mathrm{n}=104)$ or an increased dose of simvastatin $40 \mathrm{mg} /$ day $(\mathrm{n}=110)$ for 24 weeks. The results showed that there were no significant differences between treatment methods with regards to FBG, fasting plasma insulin, and A1C levels. However, LDL cholesterol levels were reduced to a greater extent in patients who received additional ezetimibe $10 \mathrm{mg}$ /day or simvastatin $20 \mathrm{mg} /$ day compared to those who received a doubled simvastatin dose (40 mg/day) (Gaudiani et al., 2005). Another study found that 2 months after the administration of combined simvastatin $10 \mathrm{mg}$ and ezetimibe $10 \mathrm{mg}$ (Vyto10) to patients with dyslipidemia, fasting insulin was significantly reduced while plasma adiponectin and insulin sensitivity were increased relative to the baseline (Koh et al., 2015).

Patients with T2DM may have multiple comorbidities that necessitate the concomitant administration of statins with other drugs. Clinical studies have shown that a combination of simvastatin with metformin and pioglitazone results in improved glycemic control (Table IV). In T2DM patients, metformin is the recommended first-line pharmacological treatment after

TABLE IV - The effect of concurrent medications in combination with simvastatin (oral antidiabetic agents)

\begin{tabular}{|c|c|c|c|c|c|c|c|c|c|c|}
\hline \multirow{2}{*}{$\begin{array}{l}\text { Concurrent } \\
\text { medication }\end{array}$} & \multirow[b]{2}{*}{ Subjects } & \multirow[b]{2}{*}{ Country } & \multirow[b]{2}{*}{$\mathbf{N}$} & \multirow{2}{*}{$\begin{array}{c}\text { Mean } \\
\text { follow-up } \\
\text { (months) }\end{array}$} & \multirow[b]{2}{*}{ Method } & \multicolumn{5}{|c|}{ Outcome } \\
\hline & & & & & & $\begin{array}{l}\text { Fasting } \\
\text { glucose }\end{array}$ & $\begin{array}{l}\text { Fasting } \\
\text { insulin }\end{array}$ & A1C & $\begin{array}{c}\text { Insulin } \\
\text { sensitivity }\end{array}$ & Adiponectin \\
\hline $\begin{array}{l}\text { Metformin } \\
\text { (Krysiak, } \\
\text { Okopien, } \\
\text { 2013b) }\end{array}$ & $\begin{array}{l}\text { IFG patients } \\
\text { treated with } \\
\text { simvastatin at } \\
\text { least } 3 \text { month }\end{array}$ & Poland & 48 & 3 & $\begin{array}{l}\text { Patient randomized } \\
\text { received MET } \\
\text { or placebo for the } \\
\text { next following } \\
90 \text { days }\end{array}$ & $\begin{array}{c}\text { MET }+ \\
\text { SIM group } \\
\text { approach near } \\
\text { significant } \\
\text { decrease FBG } \\
\text { compared } \\
\text { to before } \\
\text { randomization } \\
(p=0.071)\end{array}$ & $\begin{array}{c}\text { Not } \\
\text { measured }\end{array}$ & $\begin{array}{l}\text { MET + } \\
\text { SIM group } \\
\text { significant } \\
\text { reduce A1C } \\
\text { compared to } \\
\text { baseline and } \\
\text { placebo (p < } \\
0.001)\end{array}$ & $\begin{array}{l}\text { MET + SIM } \\
\text { group had } \\
\text { improvement } \\
\text { in HOMA-IR } \\
(\mathrm{p}<0.001)\end{array}$ & $\begin{array}{c}\text { Not } \\
\text { measured }\end{array}$ \\
\hline $\begin{array}{l}\text { Pioglitazone } \\
\text { (Forst et al., } \\
\text { 2007) }\end{array}$ & $\begin{array}{l}\text { Non-diabetic } \\
\text { patients with } \\
\text { cardiovascular } \\
\text { risk }\end{array}$ & Germany & 125 & 3 & $\begin{array}{l}\text { Patients randomizedl } \\
\text { received PIO + } \\
\text { placebo, PIO + SIM, } \\
\text { SIM + placebo. } \\
\text { Treatment started } \\
\text { with PIO } 30 \text { mg } \\
\text { or and SIM } 20 \\
\text { mg. After } 2 \text { weeks } \\
\text { increase dosage to } \\
\text { PIO } 45 \text { mg or and } \\
\text { SIM } 40 \text { mg }\end{array}$ & $\begin{array}{l}\text { AFBG reduce in } \\
\text { group treated } \\
\text { with PIO and } \\
\text { PIO + SIM }\end{array}$ & $\begin{array}{l}\text { Reduce in } \\
\text { group } \\
\text { treated with } \\
\text { PIO and } \\
\text { PIO + SIM }\end{array}$ & $\begin{array}{c}\text { Not } \\
\text { measured }\end{array}$ & $\begin{array}{l}\text { HOMA score } \\
\text { improved in } \\
\text { group treated } \\
\text { with PIO and } \\
\text { PIO + SIM }\end{array}$ & $\begin{array}{l}\text { Adiponectin } \\
\text { increase } \\
\text { in PIO and } \\
\text { PIO + SIM } \\
\text { group, while } \\
\text { reduce in } \\
\text { group treated } \\
\text { with SIM }\end{array}$ \\
\hline
\end{tabular}

Abbreviation: MET (metformin); SIM (simvastatin); PIO (pioglitazone) 
lifestyle interventions fail to result in adequate glycemic control (Rojas, Gomes, 2013). Krysiak et al. (2013b) demonstrated that metformin, when administered to simvastatin-treated patients with impaired fasting glucose levels, reduced HOMA-IR values by approximately 55\% and $\mathrm{A} 1 \mathrm{C}$ levels by 11\% (Krysiak et al., 2013b).

Pioglitazone - a thiazolidinedione that works by enhancing insulin sensitivity -improves $\mathrm{A} 1 \mathrm{C}$ levels and is beneficial in reducing free fatty acid and triglyceride levels as well as increasing HDL cholesterol (Herz et al., 2003; Kipnes et al., 2001). In a double-blinded study, pioglitazone alone and the combination of pioglitazone and simvastatin significantly improved glucose levels, insulin levels, and HOMA score. No such changes were seen in the simvastatin treatment group. In addition, it was reported that the pioglitazone-simvastatin combination was better for lowering the risk of cardiovascular events when compared to either of the drugs used alone (Forst et al., 2007).

Hypertension and hypercholesterolemia are two major health issues that contribute to increased cardiovascular disease risk (Dalal et al., 2012), and the patients are commonly treated with statins and antihypertensive agents. As shown in Table V, the effect of combined simvastatin and antihypertensive medications has been investigated, and it was found that fasting plasma

TABLE V - The effect of concurrent medications in combination with simvastatin (antihypertensive agents)

\begin{tabular}{|c|c|c|c|c|c|c|c|c|c|c|}
\hline \multirow{2}{*}{$\begin{array}{l}\text { Concurrent } \\
\text { medication }\end{array}$} & \multirow[b]{2}{*}{ Subjects } & \multirow[b]{2}{*}{ Country } & \multirow[b]{2}{*}{$\mathbf{N}$} & \multirow{2}{*}{$\begin{array}{l}\text { Mean follow- } \\
\text { up (month) }\end{array}$} & \multirow[b]{2}{*}{ Method } & \multicolumn{5}{|c|}{ Outcome } \\
\hline & & & & & & $\begin{array}{l}\text { Fasting } \\
\text { glucose }\end{array}$ & $\begin{array}{l}\text { Fasting } \\
\text { insulin }\end{array}$ & $\mathrm{A} 1 \mathrm{C}$ & $\begin{array}{c}\text { Insulin } \\
\text { sensitivity }\end{array}$ & Adiponectin \\
\hline $\begin{array}{l}\text { Perindopril or } \\
\text { barnidipine } \\
\text { (Derosa et al., } \\
\text { 2015) }\end{array}$ & $\begin{array}{l}\text { Normocholes- } \\
\text { terolemic, } \\
\text { hypertensive } \\
\text { patients with } \\
\text { nonalcoholic } \\
\text { hepatic steatosis }\end{array}$ & Italy & 149 & a & $\begin{array}{l}\text { Patients were } \\
\text { on perindopril } \\
5 \mathrm{mg} \text { /day or } \\
\text { barnidipine } \\
20 \mathrm{mg} / \text { day for } \\
6 \mathrm{months} \text { and } \\
\text { added with SIM } \\
20 \mathrm{mg} / \text { day for } \\
\text { subsequent } \\
6 \text { months }\end{array}$ & $\begin{array}{l}\text { No } \\
\text { significant } \\
\text { changes in } \\
\text { both group }\end{array}$ & $\begin{array}{l}\text { No } \\
\text { significant } \\
\text { changes in } \\
\text { both group }\end{array}$ & $\begin{array}{c}\text { Not } \\
\text { measured }\end{array}$ & $\begin{array}{c}\text { Not } \\
\text { measured }\end{array}$ & $\begin{array}{c}\text { Increase in } \\
\text { barnidipine } \\
+ \text { SIM group } \\
\text { compared to } \\
\text { baseline } \\
(\mathrm{p}<0.05)\end{array}$ \\
\hline $\begin{array}{l}\text { Losartan (Koh } \\
\text { et al., 2004) }\end{array}$ & $\begin{array}{l}\text { Hypercholes- } \\
\text { terolemia with } \\
\text { hypertensive } \\
\text { patients }\end{array}$ & Korea & 47 & $\begin{array}{c}3 \text { treatment } \\
\text { arm }(2 \text { months } \\
\text { for each }) \\
\text { and } 2 \text { washout } \\
\text { period } \\
(2 \text { months })\end{array}$ & $\begin{array}{l}\text { Patients were } \\
\text { randomized } \\
\text { receive either } \\
\text { SIM } 20 \mathrm{mg} \\
\text { + placebo, } \\
\text { SIM } 20 \mathrm{mg}+ \\
\text { losartan } 100 \mathrm{mg} \\
\text { or losartan } \\
100 \mathrm{mg}+ \\
\text { placebo daily }\end{array}$ & $\begin{array}{l}\text { No } \\
\text { significant } \\
\text { changes in } \\
\text { three group }\end{array}$ & $\begin{array}{l}\text { No } \\
\text { significant } \\
\text { changes in } \\
\text { three group }\end{array}$ & $\begin{array}{c}\text { Not } \\
\text { measured }\end{array}$ & $\begin{array}{c}\text { Losartan } \\
+ \text { SIM and } \\
\text { losartan group } \\
\text { significantly } \\
\text { increase } \\
\text { QUICKI }\end{array}$ & $\begin{array}{c}\text { Increase in } \\
\text { combination } \\
\text { losartan }+ \\
\text { SIM group } \\
(\mathrm{p}<0.001) \\
\text { and losartan } \\
\text { alone } \\
(\mathrm{p}=0.002)\end{array}$ \\
\hline $\begin{array}{l}\text { Ramipril (Koh } \\
\text { et al., 2005) }\end{array}$ & $\begin{array}{c}\text { Hypercholes- } \\
\text { terolemia with } \\
\text { T2DM }\end{array}$ & Korea & 53 & $\begin{array}{l}3 \text { treatment } \\
\text { arm }(2 \text { months } \\
\text { for each }) \\
\text { and } 2 \text { washout } \\
\text { period } \\
(2 \text { months })\end{array}$ & $\begin{array}{l}\text { Patients were } \\
\text { randomized } \\
\text { receive either } \\
\text { SIM } 20 \mathrm{mg} \\
\text { + placebo, } \\
\text { SIM } 20 \mathrm{mg}+ \\
\text { ramipril } \\
10 \mathrm{mg} \text { or } \\
\text { ramipril } \\
10 \mathrm{mg}+ \\
\text { placebo daily }\end{array}$ & $\begin{array}{l}\text { No } \\
\text { significant } \\
\text { changes in } \\
\text { three group }\end{array}$ & $\begin{array}{l}\text { No } \\
\text { significant } \\
\text { changes in } \\
\text { three group }\end{array}$ & $\begin{array}{c}\text { Not } \\
\text { measured }\end{array}$ & $\begin{array}{c}\text { Increase } \\
\text { QUICKI } \\
\text { in SIM + } \\
\text { ramipril } \\
\text { group and } \\
\text { ramipril } \\
\text { alone group }\end{array}$ & $\begin{array}{l}\text { Increase in } \\
\text { SIM + } \\
\text { ramipril } \\
\text { group and } \\
\text { ramipril } \\
\text { alone group }\end{array}$ \\
\hline $\begin{array}{l}\text { Lisinopril } \\
\text { (Kaminsky et } \\
\text { al., 2010) }\end{array}$ & $\begin{array}{l}\text { Atherosclerosis } \\
\text { and moderate } \\
\text { hypertensive }\end{array}$ & Russia & 32 & 24 & $\begin{array}{l}\text { Patients were } \\
\text { randomized } \\
\text { receive either } \\
\text { lisinopril } \\
10-20 \mathrm{mg} / \text { day } \\
\text { or lisinopril } \\
10-20 \mathrm{mg} \\
\text { added with SIM } \\
20 \mathrm{mg} \text { daily }\end{array}$ & $\begin{array}{l}\text { No } \\
\text { significant } \\
\text { changes in } \\
\text { both group }\end{array}$ & $\begin{array}{c}\text { Not } \\
\text { measured }\end{array}$ & $\begin{array}{c}\text { Not } \\
\text { measured }\end{array}$ & $\begin{array}{c}\text { Not } \\
\text { measured }\end{array}$ & $\begin{array}{c}\text { Not } \\
\text { measured }\end{array}$ \\
\hline
\end{tabular}


insulin and FBG levels were not affected by perindoprilsimvastatin or barnidipine-simvastatin regimens (Derosa et al., 2015). However, Koh et al. (2004) found that losartan alone or in combination with simvastatin resulted in a significant increase in insulin sensitivity and plasma adiponectin levels relative to the baseline, and that the difference was greater when compared to simvastatin alone (Koh et al., 2004).

\section{CONCLUSIONS}

In vitro studies have identified possible mechanisms by which simvastatin affects glucose metabolism. These include the inhibition of insulin secretion, possibly by decreasing GLUT2 activity, reducing ATP production, inhibiting L-type $\mathrm{Ca}^{2+}$ channels and decreasing cytosolic $\mathrm{Ca}^{2+}$ concentrations. Some studies have reported that simvastatin may impair glucose metabolism whereas other studies reported no effect or improvement of glucose metabolism.

Even though statins are beneficial in reducing the risk of cardiovascular events, its glycemic effect on patients should be monitored by periodically evaluating blood glucose levels regardless of whether the patients have diabetes or otherwise. Further studies are required to investigate the possible synergistic effects of statins with concurrent medication on glycemia, especially in patients with multiple comorbidities. Although the benefits of statins have been shown to outweigh its risks, it is important that glycemic control in patients is monitored for potential drug interactions between statins with the concurrent medications used. Besides that, further studies are recommended to determine whether or not the dose and duration of statin use could affect the glycemic control.

\section{ACKNOWLEDGEMENTS}

The authors would like to thank the University of Malaya for financial and technical support (Research Grant RP024C-14HTM).

\section{DECLARATION OF INTEREST STATEMENT}

The authors report no conflicts of interest.

\section{REFERENCES}

Ahmed MH, Byrne CD. Potential therapeutic uses for ezetimibe beyond lowering LDL-c to decrease cardiovascular events. Diabetes Obes Metab. 2010;12(11):958-966.
Aiman U, Najmi A, Khan RA. Statin induced diabetes and its clinical implications. J Pharmacol Pharmacother. 2014;5(3):181185.

Al-Foraih M, Somerset S. Factors affecting adherence to statins in hypercholesterolemic Kuwaiti patients: a cross-sectional study. Med Princ Pract. 2016;26(1):35-40.

Armitage J, Bowman L, Wallendszus K, Bulbulia R, Rahimi $\mathrm{K}$, Haynes R, et al. Intensive lowering of LDL cholesterol with $80 \mathrm{mg}$ versus $20 \mathrm{mg}$ simvastatin daily in 12,064 survivors of myocardial infarction: a double-blind randomised trial. Lancet. 2010;376(9753):1658-1669.

Bays H. Safety of niacin and simvastatin combination therapy. Am J Cardiol. 2008;101(8A):3B-8B.

Bellia A, Rizza S, Lombardo MF, Donadel G, Fabiano R, Andreadi K, et al. Deterioration of glucose homeostasis in type 2 diabetic patients one year after beginning of statins therapy. Atherosclerosis. 2012;223(1):197-203.

Bellia A, Rizza S, Galli A, Fabiano R, Donadel G, Lombardo $\mathrm{MF}$, et al. Early vascular and metabolic effects of rosuvastatin compared with simvastatin in patients with type 2 diabetes. Atherosclerosis. 2010;210(1):199-201.

Cannon CP. Combination therapy in the management of mixed dyslipidaemia. J Intern Med. 2008;263(4):353-365.

Ceriello A. Evidence for an Independent and Cumulative Effect of Postprandial Hypertriglyceridemia and Hyperglycemia on Endothelial Dysfunction and Oxidative Stress Generation: Effects of Short- and Long-Term Simvastatin Treatment. Circulation. 2002;106(10):1211-1218.

Chapman MJ. Fibrates in 2003: therapeutic action in atherogenic dyslipidaemia and future perspectives. Atherosclerosis. 2003;171(1):1-13.

Colhoun HM, Betteridge DJ, Durrington PN, Hitman GA, W Neil HA, Livingstone SJ, et al. Primary prevention of cardiovascular disease with atorvastatin in type 2 diabetes in the Collaborative Atorvastatin Diabetes Study (CARDS): multicentre randomised placebo-controlled trial. Lancet. 2004;364(9435):685-696.

Dalal JJ, Padmanabhan T, Jain P, Patil S, Vasnawala H, Gulati A. LIPITENSION: Interplay between dyslipidemia and hypertension. Indian J Endocrinol Metab. 2012;16(2):240. 
De Marchi U, Thevenet J, Hermant A, Dioum E, Wiederkehr A. Calcium co-regulates oxidative metabolism and ATP synthasedependent respiration in pancreatic beta cells. J Biol Chem. 2014;289(13):9182-9194.

Derosa G, Maffioli P, Salvadeo SA, Ferrari I, Gravina A, Mereu $\mathrm{R}$, et al. Fenofibrate, simvastatin and their combination in the management of dyslipidaemia in type 2 diabetic patients. Curr Med Res Opin. 2009;25(8):1973-1983.

Derosa G, Mugellini A, Pesce RM, D’Angelo A, Maffioli P. Perindopril and barnidipine alone or combined with simvastatin on hepatic steatosis and inflammatory parameters in hypertensive patients. Eur J Pharmacol. 2015;766:31-36.

Ding Y, Li Y, Wen A. Effect of niacin on lipids and glucose in patients with type 2 diabetes: A meta-analysis of randomized, controlled clinical trials. Clin Nutr. 2015;34(5):838-844.

Dobs A, Miller M, Delucca PT, Ramsey KE, Tershakovec AM, Horn W. Efficacy of simvastatin therapy in attainment of LDL-C and TG goal levels in patients with type 2 diabetic dyslipidemia. J Clin Lipidol. 2008;2(1):12-18.

Farrer M, Winocour PH, Evans K, Neil HAW, Laker MF, Kesteven P, et al. Simvastatin in non-insulin-dependent diabetes mellitus: effect on serum lipids, lipoproteins and haemostatic measures. Diabetes Res \& Clin Pract. 1994;23(2):111-119.

Food and Drug Administration. FDA. Drug Safety Communication: New restrictions, contraindications, and dose limitations for Zocor (simvastatin) to reduce the risk of muscle injury. 2011. [cited 1 February 2017]. Available from: http:// www.fda.gov/drugs/drugsafety/ucm256581.htm.

Foody JM, Joyce AT, Rudolph AE, Liu LZ, Benner JS. Cardiovascular outcomes among patients newly initiating atorvastatin or simvastatin therapy: a large database analysis of managed care plans in the United States. Clin Ther. 2008;30(1):195-205.

Forst T, Pfutzner A, Lubben G, Weber M, Marx N, Karagiannis E, et al. Effect of simvastatin and/or pioglitazone on insulin resistance, insulin secretion, adiponectin, and proinsulin levels in nondiabetic patients at cardiovascular risk--the PIOSTAT Study. Metab Clin Exp. 2007;56(4):491-496.

Furberg CD, Pitt B. Withdrawal of cerivastatin from the world market. Curr Control Trials Cardiovasc Med. 2001;2(5):205207.
Gaudiani LM, Lewin A, Meneghini L, Perevozskaya I, Plotkin D, Mitchel Y, et al. Efficacy and safety of ezetimibe co-administered with simvastatin in thiazolidinedione-treated type 2 diabetic patients. Diabetes Obes Metab. 2005;7(1):88-97.

Gazzerro P, Proto MC, Gangemi G, Malfitano AM, Ciaglia E, Pisanti S, et al. Pharmacological actions of statins: a critical appraisal in the management of cancer. Pharmacol rev. 2012;64(1):102-146.

Grover HS, Luthra S, Maroo S. Are statins really wonder drugs? J Formos Med Assoc. 2014;113(12):892-898.

Grundy SM, Vega GL, Yuan Z, Battisti WP, Brady WE, Palmisano J. Effectiveness and tolerability of simvastatin plus fenofibrate for combined hyperlipidemia (the SAFARI trial). Am J Cardiol. 2005;95(4):462-468.

Gu Q, Paulose-Ram R, Burt V, Kit B. Prescription cholesterollowering medication use in adults aged 40 and over: United States, 2003-2012. NCHS data brief, no 177. Hyattsville: National Center for Health Statistics; 2014. 2015. [cited 1 February 2017]. Available from: https://www.cdc.gov/nchs/ products/databriefs/db177.htm

Heart Protection Study Collaborative Group. MRC/BHF Heart Protection Study of cholesterol lowering with simvastatin in 20536 high-risk individuals: a randomised placebo controlled trial. Lancet. 2002;360(9326):7-22.

Heart Protection Study Collaborative Group. MRC/BHF Heart Protection Study of cholesterol-lowering with simvastatin in 5963 people with diabetes: a randomised placebo-controlled trial. Lancet. 2003;361(9374):2005-2016.

Herz M, Johns D, Reviriego J, Grossman LD, Godin C, Duran $\mathrm{S}$, et al. A randomized, double-blind, placebo-controlled, clinical trial of the effects of pioglitazone on glycemic control and dyslipidemia in oral antihyperglycemic medication-naive patients with type 2 diabetes mellitus. Clinical therapeutics. 2003;25(4):1074-1095.

Hwu C, Kwok C, Chen H, Shih K, Lee S, Hsiao L, et al. Lack of effect of simvastatin on insulin sensitivity in Type 2 diabetic patients with hypercholesterolaemia: results from a doubleblind, randomized, placebo-controlled crossover study. Diabet Med. 1999;16(9):749-754.

Hydrie MZI, Qasim R, Ahmadani MY, Miyan Z, Fawwad A, Basit A. Effect of simvastatin on insulin sensitivity in type 2 diabetic subjects. Pak J Medical Science. 2007;23(5):755. 
Igel M, Sudhop T, Bergmann K. Pharmacology of 3-Hydroxy-3Methylglutaryl-Coenzyme A Reductase Inhibitors (Statins), Including Rosuvastatin and Pitavastatin. J Clin Pharmacol. 2002;42(8):835-845.

Kaminsky Y, Suslikov A, Kosenko E. Specific and pronounced impacts of lisinopril and lisinopril plus simvastatin on erythrocyte antioxidant enzymes. J Clin Pharmacol. 2010;50(2):180-187.

Kipnes MS, Krosnick A, Rendell MS, Egan JW, Mathisen AL, Schneider RL. Pioglitazone hydrochloride in combination with sulfonylurea therapy improves glycemic control in patients with type 2 diabetes mellitus: a randomized, placebo-controlled study. Am J Med. 2001;111(1):10-17.

Koh KK, Quon MJ, Han SH, Lee Y, Kim SJ, Park JB, et al. Differential metabolic effects of pravastatin and simvastatin in hypercholesterolemic patients. Atherosclerosis. 2009;204(2):483-490.

Koh KK, Quon MJ, Han SH, Lee Y, Ahn JY, Kim SJ, et al. Simvastatin improves flow-mediated dilation but reduces adiponectin levels and insulin sensitivity in hypercholesterolemic patients. Diabetes Care. 2008;31(4):776-782.

Koh KK, Oh PC, Sakuma I, Kim EY, Lee Y, Hayashi T, et al. Vascular and metabolic effects of ezetimibe combined with simvastatin in patients with hypercholesterolemia. Int J Cardiol. 2015;199:126-131.

Koh KK, Quon MJ, Han SH, Chung W-J, Ahn JY, Seo Y-H, et al. Additive beneficial effects of losartan combined with simvastatin in the treatment of hypercholesterolemic, hypertensive patients. Circulation. 2004;110(24):3687-3692.

Koh KK, Quon MJ, Han SH, Ahn JY, Jin DK, Kim HS, et al. Vascular and metabolic effects of combined therapy with ramipril and simvastatin in patients with type 2 diabetes. Hypertension. 2005;45(6):1088-1093.

Krysiak R, Okopien B. Effect of simvastatin on hemostasis in patients with isolated hypertriglyceridemia. Pharmacology. 2013a;92(3-4):187-190.

Krysiak R, Okopien B. The effect of metformin on monocyte secretory function in simvastatin-treated patients with impaired fasting glucose. Metabolism. 2013b;62(1):39-43.
Manzoni M, Rollini M. Biosynthesis and biotechnological production of statins by filamentous fungi and application of these cholesterol-lowering drugs. Appl Microbiol Biotechnol. 2002;58(5):555-564.

Meade T, Sleight P, Collins R, Armitage J, Bowman L, Parish S, et al. Intensive lowering of LDL cholesterol with $80 \mathrm{mg}$ versus $20 \mathrm{mg}$ simvastatin daily in 12,064 survivors of myocardial infarction: a double-blind randomised trial. Lancet. 2010;376(9753):1658-1669.

National Cholesterol Education Program. Third Report of the National Cholesterol Education Program (NCEP). Expert Panel on Detection E, and Treatment of High Blood Cholesterol in Adults (Adult Treatment Panel III). Final report. Circulation. 2002;(106):3143-3421.

Paolisso G, Barbagallo M, Petrella G, Ragno E, Barbieri M, Giordano M, et al. Effects of simvastatin and atorvastatin administration on insulin resistance and respiratory quotient in aged dyslipidemic non-insulin dependent diabetic patients. Atherosclerosis. 2000;150(1):121-127.

Pedersen TR, Olsson AG, F rgeman O, Kjekshus J, Wedel $\mathrm{H}$, Berg K, et al. Lipoprotein Changes and Reduction in the Incidence of Major Coronary Heart Disease Events in the Scandinavian Simvastatin Survival Study (4S). Circulation. 1998;97(15):1453-1460.

Perreault S, Ellia L, Dragomir A, Cote R, Blais L, Berard A, et al. Effect of statin adherence on cerebrovascular disease in primary prevention. Am J Med. 2009;122(7):647-655.

Rojas L, Gomes MB. Metformin: an old but still the best treatment for type 2 diabetes. Diabetol Metab Syndr. 2013;5(1):6.

Sazonov V, Maccubbin D, Sisk CM, Canner P. Effects of niacin on the incidence of new onset diabetes and cardiovascular events in patients with normoglycaemia and impaired fasting glucose. Int J Clin Pract. 2013;67(4):297-302.

Schachter M. Chemical, pharmacokinetic and pharmacodynamic properties of statins: an update. Fundam Clin Pharmacol. 2005;19(1):117-125.

Sen K, Misra A, Kumar A, Pandey RM. Simvastatin retards progression of retinopathy in diabetic patients with hypercholesterolemia. Diabetes Res Clin Pract. 2002;56(1):111. 
Sirtori CR. The pharmacology of statins. Pharmacol Res. 2014;88:3-11.

Szendroedi J, Anderwald C, Krssak M, Bayerle-Eder M, Esterbauer H, Pfeiler G, et al. Effects of high-dose simvastatin therapy on glucose metabolism and ectopic lipid deposition in nonobese type 2 diabetic patients. Diabetes Care. 2009;32(2):209-214.

Tsutamoto T, Yamaji M, Kawahara C, Nishiyama K, Fujii M, Yamamoto T, et al. Effect of simvastatin vs. rosuvastatin on adiponectin and haemoglobin A1c levels in patients with non-ischaemic chronic heart failure. Eur J Heart Fail. 2009;11(12):1195-1201.

Vijan S, Hayward RA. Pharmacologic lipid-lowering therapy in type 2 diabetes mellitus: background paper for the American College of Physicians. Ann Intern Medic. 2004;140(8):650-658.

Vittone F, Chait A, Morse JS, Fish B, Brown BG, Zhao XQ. Niacin plus Simvastatin reduces coronary stenosis progression among patients with metabolic syndrome despite a modest increase in insulin resistance: a subgroup analysis of the HDL-Atherosclerosis treatment study (HATS). J Clin Lipidol. 2007;1(3):203-210.
Yada T, Nakata M, Shiraishi T, Kakei M. Inhibition by simvastatin, but not pravastatin, of glucose-induced cytosolic $\mathrm{Ca} 2+$ signalling and insulin secretion due to blockade of $\mathrm{L}$-type $\mathrm{Ca} 2+$ channels in rat islet $\beta$-cells. Br J Pharmacol. 1999;126(5):1205-1213.

Zhao X-Q, Morse JS, Dowdy AA, Heise N, DeAngelis D, Frohlich J, et al. Safety and tolerability of simvastatin plus niacin in patients with coronary artery disease and low high-density lipoprotein cholesterol (The HDL Atherosclerosis Treatment Study). Am J Cardiol. 2004;93(3):307-312.

Zhou J, Li W, Xie Q, Hou Y, Zhan S, Yang X, et al. Effects of simvastatin on glucose metabolism in mouse MIN6 cells. J Diabetes Res. 2014;2014:376570.

Received for publication on $03^{\text {rd }}$ April 2017 Accepted for publication on $30^{\text {th }}$ August 2017 